\title{
Cribriform Adenocarcinoma of the Tongue and Minor Salivary Gland: Transoral Robotic Surgical Resection
}

\author{
Douglas M. Worrall Jason A. Brant Raymond L. Chai \\ Gregory S. Weinstein \\ Department of Otorhinolaryngology - Head and Neck Surgery, University of Pennsylvania \\ Medical Center, Philadelphia, Pa., USA
}

\section{Key Words}

Minor salivary gland cancer · Tonsillar surgery · Minimally invasive surgery · da Vinci surgical system · Transoral robotic Surgery

\begin{abstract}
Background/Aims: Cribriform adenocarcinoma of the tongue and minor salivary gland (CATMSG) is a rare, locally invasive, and poorly recognized tumor, typically occurring on the base of the tongue. This case report describes the previously unreported use of transoral robotic surgery (TORS) for the local resection of CATMSG in a novel location, the palatine tonsil, and leverages follow-up information to compare TORS to conventional surgical approaches. Methods: We performed transoral radical tonsillectomy, limited pharyngectomy, and baseof-tongue resection with staged left selective neck dissection. Results: Tumor pathology revealed an infiltrating salivary gland carcinoma with perineural invasion and a histologically similar adenocarcinoma in 1 of 64 left neck lymph nodes. TORS was performed with no perioperative complications, and the patient was subsequently discharge on postoperative day 3 with a Dobhoff tube. Postoperatively, the Dobhoff tube was removed at 1 month, the patient was advanced to soft foods by mouth at 2 months, and 3-month positron emission tomography-computed tomography scan showed no evidence of distant metastases and evolving postsurgical changes in the left tonsillectomy bed. Conclusions: This case report highlights the use of TORS resection with minimal acute and long-term morbidity compared to conventional approaches for the resection of this rare, locally invasive salivary gland carcinoma in the palatine tonsil.




\section{Introduction}

Cribriform adenocarcinoma of the tongue and minor salivary gland (CATMSG) was originally described in 1999 [1, 2] as divergent from polymorphous low-grade adenocarcinoma (PLGA). It differs from PLGA in location of the primary, cytology, overarching tumor architecture, local behavior, and tendency for early nodal metastatic disease [3]. Of 31 published cases, CATMSG occurred on the tongue in 21 patients. It was also found on the soft palate, retromolar trigone, upper lip, floor of the mouth, and lingual tonsils [3-7]. Of the 31 published cases, 19 had synchronous cervical lymph node metastases. Histologically, CATMSG is different from PLGA and papillary thyroid carcinoma (PTC) because of its distinct pale overlapping nuclei growing in solid, papillary, and cribriform architectures. These tumors consistently stain positive for cytokeratin markers, smooth muscle actin, and S-100 protein but are negative for thyroglobulin and TTF-1 [4, 5, 8]. Transoral robotic surgery (TORS) offers excellent visualization and access for resection of tumors of the oro- and hypopharynx, allowing for complete resection with negative margins, sparing patients from highly morbid open procedures with longer hospital stays and increased complications [9-17]. This case report highlights the novel use of TORS for excision of this rare variant of CATMSG in the palatine tonsil.

\section{Case Report}

A 62-year-old female with coronary artery disease, Raynaud's disease, migraine, and hypertension was referred from an outside specialist for management of a left tonsillar mass. Upon initial presentation, she had a smooth, firm, nonulcerated, $1.5-\mathrm{cm}$ lesion originating from the left tonsillar pillar. She underwent an enhanced neck computed tomography (CT) scan showing heterogeneous enhancement in the region of the left tonsillar fossa extending to the glossotonsillar sulcus that was concerning for malignancy. There was no evidence of extension into the pterygoid musculature, prevertebral space, or nasopharynx. No pathologic cervical lymph nodes were identified by imaging criteria. The patient was then taken to the operating room at an outside institution for transoral biopsy. The final pathology was initially read as PLGA of the minor salivary gland with a tumor present at the deep margin. She then underwent a positron emission tomography-CT (PET/CT) scan revealing a fluorodeoxyglucose (FDG)-avid left tonsillar focus, due to either residual disease or postsurgical change (fig. 1), and a mildly FDG-avid left level IIa cervical node without evidence of distant metastases. She was then referred to the Hospital of the University of Pennsylvania for further management.

The original pathology was re-reviewed at our institution, revealing an infiltrating salivary gland carcinoma with focal perineural invasion organized in sheets, nests, and tubules with papillary architecture. The tumor stained positive for S100 and CK7 and negative for p63, calponin, TTF-1, and thyroglobulin, making it consistent with CATMSG and atypical for PLGA. She was then scheduled for a left selective neck dissection followed by TORS for definitive management. Neck dissection pathology results demonstrated metastatic adenocarcinoma in 1 out of 64 lymph nodes that were histologically similar to the primary lesion as expected with CATMSG. There was no evidence of extracapsular extension or submandibular gland involvement. After appropriate positioning of the patient and the da Vinci ${ }^{\circledR}$ Si surgical robot (Intuitive Surgical, Sunnyvale, Calif., USA), a transoral view of the left palatine tonsil was visualized (fig. 2a), and the resection outline (fig. $2 \mathrm{~b}$ ) was created using electrocautery.

A transoral radical tonsillectomy, limited pharyngectomy, and base-of-tongue resection was performed as previously described [13]. Briefly, an incision through the buccal mucosa at the level of the pterygomandibular raphe between the upper and lower molars was made. A plane was developed along the lateral aspect of the constrictor muscles and continued laterally until the medial pterygoid musculature was identified (fig. 2c). The soft palate was then transected, and constrictor muscles were elevated off the prevertebral fascia using blunt dissection. The carotid arterial system was identified with the assistance of preoperative scans and numerous transverse veins, and arteries were clipped while small vessels were cauterized. Attention was then turned to achieving a negative margin at the base of the tongue. The base of the tongue was resected to the level of the vallecula and a final transection was made at the junction of the tongue base, 
Fig. 1. Axial fused PET/CT image demonstrating asymmetrically increased FDG focus in the left tonsillar pillar with associated soft tissue prominence, concerning for residual disease after transoral biopsy.

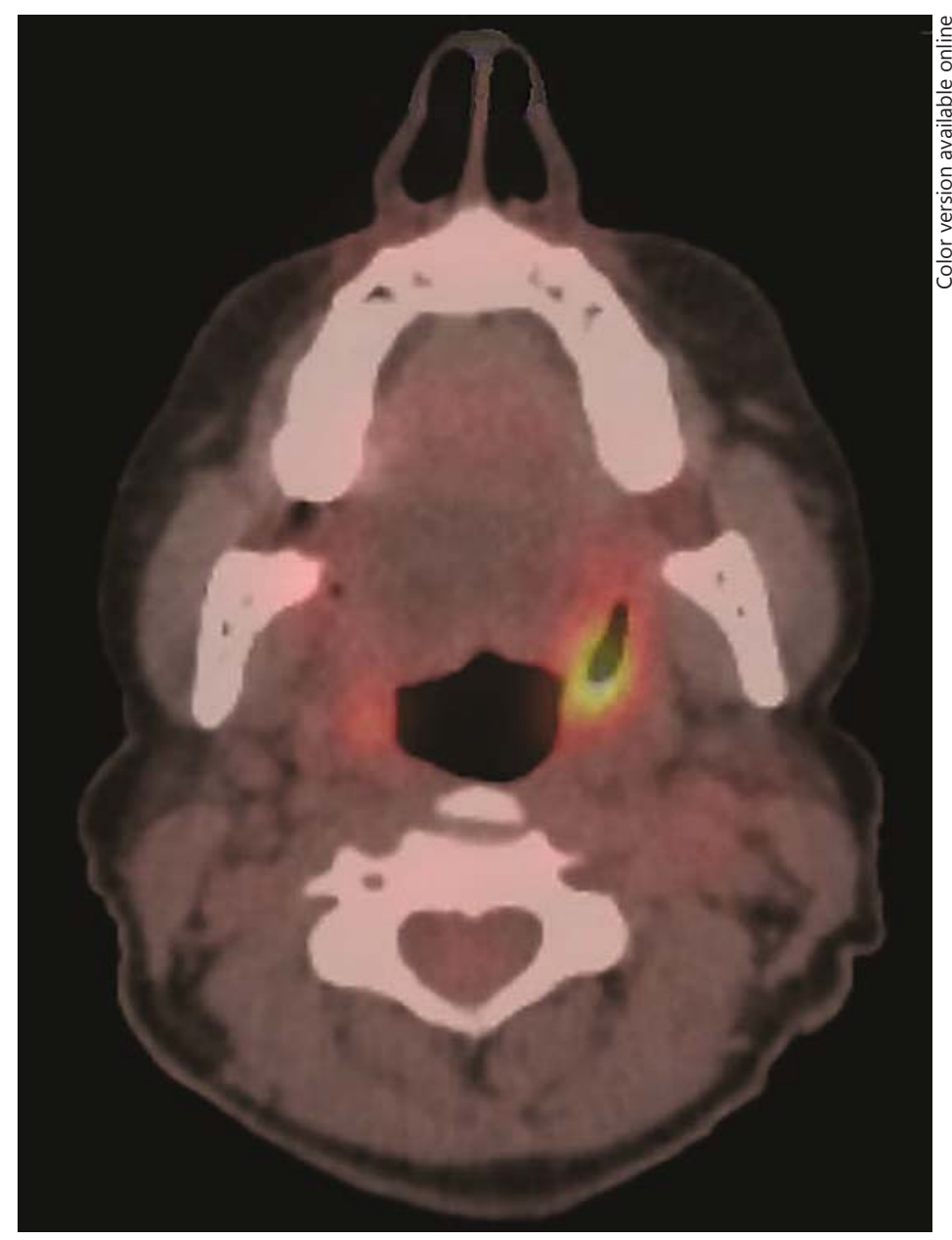

extrinsic musculature, and posterior pharyngeal wall. After the specimen was removed and oriented for pathology, the remaining area was inspected and hemostasis was obtained (fig. $2 \mathrm{~d}$ ). Following resection, the patient had a nasogastric tube placed and was monitored as an inpatient for 3 days. She was able to tolerate oral feeds on postoperative day 2 following a speech and swallow evaluation. She had minimal pain and no complications in the short postoperative period and was discharged home on postoperative day 3 . The final pathology revealed negative margins. There were no perioperative complications.

At the 2-week follow-up, the patient reported feeling well with only mild numbness of the neck and dysphagia. Flexible laryngoscopy of the nasopharynx and oropharynx showed no evidence of a tumor. Follow-up was initiated for dysphagia management, and the Dobhoff tube was removed approximately 1 month following TORS resection when she was able to tolerate thick and thin liquids by mouth. Two months postoperatively, the patient was tolerating soft, moist foods by mouth, and the 3-month PET/CT scan showed no evidence of distant metastases and evolving postsurgical changes in the left tonsillectomy bed.

\section{Discussion}

This case report illustrates the previously unreported use of TORS for the resection of a cribriform adenocarcinoma of the palatine tonsil. As demonstrated in this particular case, CATMSG is frequently misdiagnosed as PLGA or metastatic PTC [3-5]. In fact, the few documented cases of PLGA with regional lymph node metastases may actually represent CATMSG 

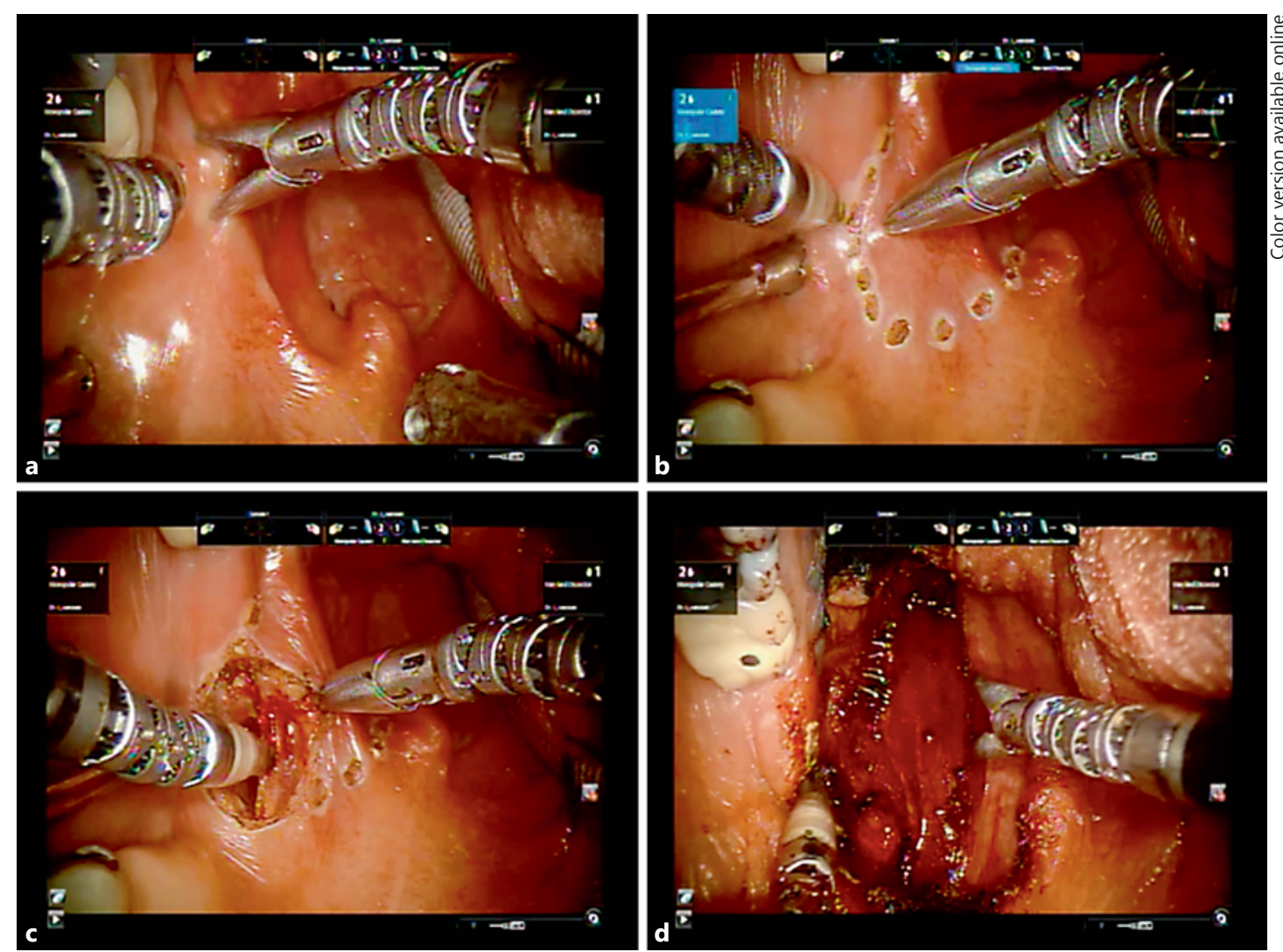

Fig. 2. a TORS visualization of the left palatine tonsil. b Outlined area of resection. c Lateral border in the parapharyngeal space. $\mathbf{d}$ Transoral view after resection.

based on its distinct histology $[1,3,8]$. PLGA has characteristic target-like concentric whorls and single-file columns of cells [2], while CATMSG has pale overlapping nuclei of cells that proliferate in solid, cribriform, and papillary architectures amidst a fibrous stroma. CATMSG occasionally has psammoma bodies, exacerbating the potential for misdiagnosis as metastatic PTC, which has led to unnecessary thyroidectomies. However, CATMSG lacks the cysts and deeply eosinophilic colloid associated with PTC and reliably stains negative for TTF-1 and thyroglobulin markers and positive for cytokeratin, smooth muscle actin, and S-100 protein $[4,5]$. Furthermore, CATMSG classically has an intact mucosa devoid of ulceration or dysplasia, with a tendency to invade local musculature and adjacent tissues, making initial resection by conventional means difficult $[3,4,6,7,12-14]$. This difficulty was evident in our case, where initial resection failed to obtain negative margins. Additionally, this case supports the current literature, highlighting that this disease predominantly affects the middle-aged, and the majority have cervical lymph node metastases at the time of diagnosis with identical histology to the primary tumor. Furthermore, although frequently located at the base of the tongue, this case illustrates that this disease can manifest in other areas of the oral cavity and represents the only documented case occurring in the palatine tonsil $[3,4]$. In addition to the rarity of and little knowledge about this newly described neoplasm, this case report exemplifies how TORS can be employed for excellent local control with minimal morbidity.

Since 2006, the literature surrounding TORS has rapidly expanded. In general, robotic surgery has gained support across many fields due to the benefits of magnification, 3D visualization, tremor filtration, and instrument stabilization [9]. TORS using the da Vinci surgical 
Worrall et al.: Cribriform Adenocarcinoma of the Tongue and Minor Salivary Gland: Transoral Robotic Surgical Resection

system for oropharyngeal carcinoma resection offers well-documented local control with or without adjuvant therapy [18], with decreased postoperative complications, decreased longterm morbidity, and shorter operative times and hospital stays compared to conventional transoral or open approaches [10-18]. Furthermore, when examining the quality of life, patients treated with TORS alone had a return to baseline functional status and overall quality of life at 12 months with only a modest impact on speech at the 6- and 12-month follow-ups [19]. This is evident by our patient's rapid return to oral intake and discharge to her home on postoperative day 3 and removal of the Dobhoff tube at 1 month postoperatively. All previously documented cases of CATMSG were treated with nonrobotic radical or simple transoral excision, many received neck lymph node dissections, and 14 received adjuvant radiation therapy. Simple excision is fraught with positive margins and recurrences while radical resection offers improved local control at the expense of significant functional deficits. In the past, open mandibulectomy and subsequent free-flap reconstruction were occasionally required when visualization of the tumor was suboptimal $[12,13]$. However, TORS, with its 3D high-definition vision system and lack of line-of-sight limitations, provides excellent visualization, allowing for superior resection with low rates of positive margins and minimal morbidity, which makes it, along with neck dissection when indicated, an ideal surgical intervention for CATMSG.

\section{Conclusion}

The case presented here highlights the identification of this rare, poorly recognized tumor in an unusual location, the palatine tonsil, with synchronous nodal metastases by histopathologic characteristics and immunohistochemistry profile. Furthermore, it demonstrates the utilization of TORS, leveraging the superior visualization to obtain excellent local control with minimal acute and long-term morbidity in comparison to conventional transoral surgical approaches.

\section{References}

1 Michal M, Skalova A, Simpson RH, Raslan WF, Curik R, Leivo I, Mukensnabl P: Cribriform adenocarcinoma of the tongue: a hitherto unrecognized type of adenocarcinoma characteristically occurring in the tongue. Histopathology 1999;35:495-501.

2 Luna MA, Wenig BM: Polymorphous low grade adenocarcinoma; in Barnes L, Eveson JW, Reichart P, Sidransky D (eds): World Health Organization Classification of Tumours: Pathology and Genetics of Head and Neck Tumours. Lyon, IARC Press, 2005.

3 Michal M, Kacerovska D, Kazakov DV: Cribriform adenocarcinoma of the tongue and minor salivary glands: a review. Head Neck Pathol 2013;7:S3-S11.

4 Skalova A, Sima R, Kaspirkova-Nemcova J, Simpson RHW, Elmberger G, Levivo I, Di Palma S, Jirasek T, Gnepp DR, Weinreb I, Perez-Ordonez B, Mukensanbl P, Rychly B, Hrabal P, Michal M: Cribriform adenocarcinoma of minor salivary gland origin principally affecting the tongue: characterization of new entity. Am J Surg Pathol 2011;35:1168-1176.

5 Laco J, Kamardova K, Vitkova P, Sehnalkova E, Dvorakova S, Vacalavikova E, Sykorova V, Kaspirkova J, Skalova A, Ryska A: Cribriform adenocarcinoma of minor salivary glands may express galectin-3, cytokeratin 19, and HMBE-1 and contains polymorphisms of RET and H-RAS proto-oncogenes. Virchows Arch 2012;461:531540.

6 Cocek A, Hronkova K, Voldanova J, Sach J, Sklova A, Ambrus M, Vranova J, Hahn A: Cribriform adenocarcinoma of the base of the tongue and low-grade, polymorphic adenocarcinomas of the salivary glands. Oncol Lett 2011;2:135-138.

7 Borowski-Borowy P, Dyduch G, Papla B, Gabrys I, Skladzien J, Okon K: Cribriform adenocarcinoma of the tongue. Pol J Pathol 2011;3:168-171.

8 Gailey MP, Bayon R, Robinson RA: Cribriform adenocarcinoma of minor salivary gland: a report of two cases with emphasis on cytology. Diagn Cytopathol 2014;42:1085-1090. 
Worrall et al.: Cribriform Adenocarcinoma of the Tongue and Minor Salivary Gland: Transoral Robotic Surgical Resection

9 Herron DM, Marohn M; Sages-MIRA Robotic Surgery Consensus Group: A consensus document on robotic surgery. Surg Endosc 2008;22:313-325.

10 O'Malley BW Jr, Weinstein GS, Synder W, Hockstein NG: Transoral robotic surgery (TORS) for base of tongue neoplasms. Laryngoscope 2006;116:1465-1472.

11 Weinstein GS, O'Malley BW Jr, Cohen MA, Quon H: Transoral robotic surgery for advanced oropharyngeal carcinoma. Arch Otolaryngol Head Neck Surg 2010;136:1079-1085.

12 Poulsen M, Porceddu SV, Kingsley PA, Tripcon L, Coman W: Locally advanced tonsillar squamous cell carcinoma: treatment approach revisited. Laryngoscope 2007;117:45-50.

13 Weinstein GS, O’Malley BW Jr, Synder W, Sherman E, Quon H: Transoral robotic surgery radical tonsillectomy. Arch Otolaryngol Head Neck Surg 2007;133:1220-1226.

14 Weinstein GS, O'Malley BW Jr, Desai SC, Quon H: Transoral robotic surgery: does the end justify the means? Curr Opin Otolaryngol Head Neck Surg 2009;17:126-131.

15 Moore EJ, Olsen KD, Kasperbauer JL, Hinni ML, Salassa JR: Transoral robotic surgical extirpation of oropharyngeal neoplasms. 7th Int Conf Head Neck Cancer, San Francisco, 2008.

16 Boudreaux BA, Rosenthal EL, Magnuson JS, Clemons LK, Newman JR, Carroll WR: Feasibility and safety of robotic surgery for upper aerodigestive tract neoplasms. 7th Int Conf Head Neck Cancer, San Francisco, 2008.

17 Machtay M, Perch S, Markiewicz D, Thaler E, Chalian A, Goldberg A, Kligerman M, Weinstein G: Combined surgery and postoperative radiotherapy for carcinoma of the base of radiotherapy for carcinoma of the base of tongue: analysis of treatment outcome and prognostic value of margin status. Head Neck 1997;19:494-499.

18 Weinstein GS, Quon H, Newman J, Chalian A, Malloy K, Lin A, Desai A, Livolsi VA, Montone KT, Cohen KR, O’Malley BW Jr: Transoral robotic surgery alone for oropharyngeal cancer - an analysis of local control. Arch Otolaryngol Head Neck Surg 2012;138:628-634.

19 LeonhardtFD, Quon H, Abrahao M, O'Malley BW Jr, Weinstein GS: Transoral robotic surgery for oropharyngeal carcinoma and its impact on patient-reported quality of life and function. Head Neck 2012;34:146-154. 